\title{
Structures on Fuzzy Groups and L- Fuzzy Number
}

\author{
R.Nagarajan \\ Assistant Professor \\ Department of Mathematics \\ $\mathrm{J} J$ College of Engineering \& Technology \\ Tiruchirappalli- 620009, Tamilnadu, India
}

\author{
A.Solairaju \\ Associate Professor \\ PG \& Research Department of Mathematics \\ Jamal Mohamed College \\ Tiruchirappalli- 620020, Tamilnadu, India
}

\begin{abstract}
The theory of fuzzy sets has developed in many directions and is finding applications in a wide variety of fields. Resentful in 1971 used this concept to develop the theory of fuzzy groups in this paper we have given independent proof of several theorems on fuzzy groups. We discuss about Fuzzy groups and investigate some of their structures on the concept of Fuzzy Group family and L- fuzzy number.
\end{abstract}

Keyword: Fuzzy sets, fuzzy groups, fuzzy group family, standardized fuzzy group, L- fuzzy number

AMS 2000 Subject classification: 20N25, 03E72, 08A72.

\section{INTROUDUCTION}

Since the paper fuzzy set theory has been considerably developed by Zadeh himself and some researchers. The original concept of fuzzy sets was introduced as an extension of cribs (usual) sets, by enlarging the truth value set of "grade of members" from the two value set $\{0,1\}$ to unit interval $\{0,1\}$ of real numbers. The basic concepts of fuzzy set theory are in $[2,3,5,6,7,8]$.The study of fuzzy group was started by Rosenfeld. It was extended by Roventa who have introduced the fuzzy groups operating on fuzzy sets. Several mathematicians have followed them in investigating the fuzzy group theory in $[1,3,4,5,9]$. We now recall the previous and preliminary definitions, and results that are required in our discussion.

\section{SOME PROPERTIES ON FUZZY GROUPS}

2.1Proposition: Let $\mathrm{G}$ be a fuzzy group and $\hat{\mathrm{S}}$ be. a fuzzy subset of Then $\hat{S}$ is a fuzzy subgroup of $G$ ( written $\leq$ ) iff $\mu\left(\mathrm{xy}^{-1}\right) \geq \min \{\mu(\mathrm{x}), \mu(\mathrm{y})\}$ for all $\mathrm{x}, \mathrm{y}$ in $\mathrm{S}$.

Satisfied in. Therefore we obtain

$\mu\left(x^{-1}\right) \geq \min \left\{\mu(x), \mu\left(y^{-1}\right)\right\}$

$$
=\min \{\mu(\mathrm{x}), \mu(\mathrm{y})\}
$$

Case (ii): Let $\mu\left(x y^{-1}\right) \geq \min \{\mu(x), \mu(y)\}$ for all $x, y$ $\varepsilon S$

If $y=x$ in $(1)$, we have $\mu_{\hat{S}}\left(x^{-1}\right)=\mu_{\hat{S}}(e) \geq \min \left\{\mu_{\hat{S}}(x)\right.$, $\left.\mu_{\hat{\mathrm{S}}}(\mathrm{x})\right\}=\mu_{\hat{\mathrm{S}}}(\mathrm{x})$

If $\mathrm{x}=\mathrm{e}$ in $(1), \mu_{\hat{\mathrm{S}}}\left(\mathrm{y}^{-1}\right)=\mu_{\hat{\mathrm{S}}}\left(\mathrm{ey} \mathrm{y}^{-1}\right) \geq \min \left\{\mu_{\hat{\mathrm{S}}}(\mathrm{e})\right.$, $\left.\mu_{\hat{\mathrm{S}}}(\mathrm{x})\right\}=\mu_{\hat{\mathrm{S}}}(\mathrm{y})$, for all y $\varepsilon \mathrm{S}$

Implies that $\mu_{\hat{\mathrm{S}}}(\mathrm{y}) \geq \mu_{\hat{\mathrm{S}}}\left(\mathrm{y}^{-1}\right)$ for all y $\varepsilon$ S. (since substituting $\mathrm{y}=\mathrm{y}^{-1}$ in the above equation). Therefore $\mu_{\hat{\mathrm{S}}}(\mathrm{y})=\mu_{\hat{\mathrm{S}}}\left(\mathrm{y}^{-1}\right)$. (FG2) is satisfied in $\hat{\mathrm{S}}$.

Furthur, $\mu_{\hat{\mathrm{s}}}\left(\mathrm{x}\left(\mathrm{y}^{-1}\right)^{-1} \geq \min \left\{\mu_{\hat{\mathrm{s}}}(\mathrm{x}), \mu_{\hat{\mathrm{s}}}\left(\mathrm{y}^{-1}\right)\right\}\right.$

$$
=\min \left\{\mu_{\hat{\mathrm{S}}}(\mathrm{x}), \mu_{\hat{\mathrm{S}}}(\mathrm{y})\right\} \mathrm{FG} 1 \text { is }
$$

satisfied in $\hat{\mathrm{S}}$.

2.2 Lemma: For all $a, b \varepsilon(0,1)$ and $m$ is any positive integer, verify that
i) If $a<b$ then $a^{m}<b^{m}$ and
ii) $\min \{a, b\}=\min \left\{a^{m}, b^{m}\right\}$.

Proof: It is obvious. 
2.3 Proposition: If $G$ is a group, then prove that $\mathrm{G}^{\mathrm{m}}=\left\{\left(\mathrm{x}, \mu_{\mathrm{G}}(\mathrm{x})^{\mathrm{m}}\right) / \mathrm{x} \varepsilon \mathrm{G}\right\}$ is a fuzzy group.

Proof: Let $G$ is a fuzzy group, where $(G, \bullet)$ is a group. Thus $\left(\mathrm{G}^{\mathrm{m}}, \bullet\right)$ is a group for all the positive integer $\mathrm{m}$. Let $\mathrm{m}$ is a positive integer and $\mathrm{x}, \mathrm{y} \varepsilon \mathrm{G}^{\mathrm{m}}$.

Claim: $\left(\mu_{\mathrm{G}} \mathrm{m}(\mathrm{xy})\right) \geq \min \left\{\mu_{\mathrm{G}} \mathrm{m}(\mathrm{x}), \mu_{\mathrm{G}} \mathrm{m}(\mathrm{y})\right\}$

$$
\begin{aligned}
(\mathrm{FG} 1)\left(\mu_{\mathrm{G}} \mathrm{m}(\mathrm{xy})\right) & =\left(\mu_{\mathrm{G}}(\mathrm{xy})\right)^{\mathrm{m}} \\
\geq & \min \left\{\left(\mu_{\mathrm{G}}(\mathrm{x}), \mu_{\mathrm{G}}(\mathrm{y})\right\}^{\mathrm{m}}\right. \\
= & \min \left\{\left(\mu_{\mathrm{G}}(\mathrm{x})\right)^{\mathrm{m}},\left(\mu_{\mathrm{G}}(\mathrm{y})\right)^{\mathrm{m}}\right\} \quad(\mathrm{FG} 1 \text { is }
\end{aligned}
$$

satisfied in G.)

$$
\geq \min \left\{\left(\mu_{\mathrm{G}} \mathrm{m}(\mathrm{x}), \mu_{\mathrm{G}} \mathrm{m}(\mathrm{y})\right\}\right.
$$

FG1 is satisfied in $\mathrm{G}^{\mathrm{m}}$.

$$
\text { (ii) } \begin{aligned}
\mu_{\mathrm{G}} \mathrm{m}(\mathrm{x}) & =\left(\mu_{\mathrm{G}}(\mathrm{x})\right)^{\mathrm{m}} \\
& =\left(\mu_{\mathrm{G}}\left(\mathrm{x}^{-1}\right)\right)^{\mathrm{m}} \text { since }(\mathrm{FG} 2) \text { is satisfied }
\end{aligned}
$$

in $\mathrm{G}$.

$$
=\left(\mu_{\mathrm{G}} \mathrm{m}\left(\mathrm{x}^{-1}\right)\right.
$$

$\left(\mathrm{G}^{\mathrm{m}}, \bullet\right)$ is a fuzzy group by (1.7).

(iii) $\mu_{\mathrm{G}} \mathrm{m}(\mathrm{e})=\left(\mu_{\mathrm{G}}(\mathrm{e})\right)^{\mathrm{m}}=(1)^{\mathrm{m}}=1$.

Hence $\left(\mathrm{G}^{\mathrm{m}}, \bullet\right)$ forms a standardized fuzzy group.

2.4 Corollary : The fuzzy group $G^{n}$ is a fuzzy subgroup of $\mathrm{G}^{\mathrm{m}}$, if $\mathrm{m} \leq \mathrm{n}$.

Proof: Clearly $G^{\mathrm{n}}$ and $\mathrm{G}^{\mathrm{m}}$ are fuzzy groups by (2.3). For all $\mathrm{x} \varepsilon[0,1], \mathrm{x}^{\mathrm{m}} \geq \mathrm{x}^{\mathrm{n}}$ implies that $\mathrm{G}^{\mathrm{n}} \mathrm{C} \mathrm{G}^{\mathrm{m}}$ ( since $\mu_{\mathrm{G}} \mathrm{n}(\mathrm{x}) \leq \mu_{\mathrm{G}} \mathrm{m}(\mathrm{x})$ for all $\mathrm{x} \varepsilon \mathrm{G}$ ).

2.5 Proposition : If $\hat{G}^{i}$ and $\hat{G}^{j}$ are fuzzy groups, then $\hat{\mathrm{G}}^{\mathrm{i}} \mathrm{U} \hat{\mathrm{G}}^{\mathrm{j}}$ is also a fuzzy group if $\mathrm{i}<\mathrm{j}$

Proof : since $\mathrm{i}<\mathrm{j}$, then we have

$$
\begin{aligned}
& \mu_{\hat{G}} \mu^{i}>\mu_{\hat{G}} \mu^{j} \\
& \text { (FGI) } \mu_{\hat{G i} \mathrm{U} \hat{\mathrm{G} j}}{ }^{(\mathrm{xy})}=\max \left\{\mu_{\hat{\mathrm{G}}}^{\mathrm{i}}(\mathrm{xy}), \mu_{\mathrm{G}}^{\mathrm{j}}(\mathrm{xy})\right\} \\
& =\max \left\{\left(\mu_{\hat{G}}(x y)\right)^{i},\left(\mu_{\hat{G}}(x y)\right)^{j}\right\} \\
& =\left(\mu_{\hat{\mathrm{G}}}(\mathrm{xy})\right)^{\mathrm{i}} \\
& \geq \min \left\{\mu_{\hat{G}}^{i}(x), \mu_{\hat{G}}^{i}(y)\right\} \\
& \geq \min \left\{\max \left\{\mu_{\hat{\mathrm{G}}}^{\mathrm{i}}(\mathrm{x}), \mu_{\hat{\mathrm{G}}}^{\mathrm{i}}(\mathrm{y})\right\}, \max \left\{\mu_{\hat{\mathrm{G}}}^{\mathrm{i}}(\mathrm{x}), \mu_{\mathrm{G}}^{\mathrm{j}}(\mathrm{y})\right\}\right\} \\
& \geq \min \left\{\max \left\{\mu_{\mathrm{G}}^{\mathrm{i}}(\mathrm{x}), \mu_{\mathrm{G}_{\mathrm{G}}^{\mathrm{j}}}^{\mathrm{j}}(\mathrm{x})\right\}, \max \left\{\mu_{\hat{\mathrm{G}}}^{\mathrm{i}}(\mathrm{y}), \mu_{\hat{\mathrm{G}}}^{\mathrm{i}}(\mathrm{y})\right\}\right\} \\
& \geq \min \left\{\mu_{\hat{G} i U G \hat{j}}(x), \mu_{\hat{G i U G ̂ j}}(y)\right\}
\end{aligned}
$$

FGI is satisfied.

$(\mathrm{FG} 2) \mu_{\hat{G i} \mathrm{U}} \hat{G}_{\mathrm{j}}(\mathrm{x})=\max \left\{\mu_{\hat{G i}}(\mathrm{x}), \mu_{\hat{G i}}(\mathrm{x})\right\}$

$$
\begin{aligned}
& =\max \left\{\mu_{\hat{G}}(x)^{i}, \mu_{\hat{G}}(x)^{j}\right\} \\
& =\max \left\{\mu_{\hat{G}}\left(x^{-1}\right)^{i}, \mu_{\hat{G}}\left(x^{-1}\right)^{j}\right\}
\end{aligned}
$$

Volume 6-No.3, September 2010 $=\max \left\{\mu_{\mathrm{Gi}}\left(\mathrm{x}^{-1}\right), \mu_{\hat{\mathrm{G} j}}\left(\mathrm{x}^{-1}\right)\right\}$

$$
=\mu_{\hat{G i U} \hat{\mathrm{G} j}}\left(\mathrm{x}^{-1}\right)
$$

FG2 is satisfied.

$$
\begin{aligned}
& (\mathrm{FG} 3) \mu_{\hat{G i} \mathrm{U} \hat{\mathrm{G} j}}(\mathrm{e})=\max \left\{\mu_{\hat{\mathrm{G} i}}(\mathrm{e}) \mu_{\hat{\mathrm{G}} \mathrm{j}}(\mathrm{e})\right\} \\
& \qquad=\max \{1,1\}(\hat{\mathrm{G}} \text { is a fuzzy } \\
& \text { group). } \\
& \qquad \hat{\mathrm{G}}^{\mathrm{i}} \mathrm{U} \hat{\mathrm{G}}^{\mathrm{j}} \text { forms a fuzzy group. }
\end{aligned}
$$

2.6 Proposition : $\hat{G}^{i}$ and $\hat{G}^{j}$ are fuzzy groups then $\hat{\mathrm{G}}^{\mathrm{i}} \cap \hat{\mathrm{G}}^{\mathrm{j}}$ also a fuzzy group if $\mathrm{i}<\mathrm{j}$

Proof : since $\mathrm{i}<\mathrm{j}$

$$
\begin{aligned}
& \text { (FGI) } \mu_{\hat{G i} \cap \hat{G j}}\left(x=\min \left\{\mu_{\hat{G i}}(x y), \mu_{\hat{G j}}(x y)\right\}\right. \\
& =\min \left\{\left(\mu_{\hat{\mathrm{G}}}(\mathrm{xy})\right)^{\mathrm{i}},\left(\mu_{\hat{\mathrm{G}}}(\mathrm{xy})\right)^{\mathrm{j}}\right\} \\
& =\left(\mu_{\hat{\mathrm{G}}}(\mathrm{xy})\right)^{\mathrm{i}} \\
& \geq \min \left\{\mu_{\hat{\mathrm{G} j}}(\mathrm{x}), \mu_{\hat{\mathrm{G}} \mathrm{j}}(\mathrm{y})^{\mathrm{j}}\right\} \\
& =\min \left\{\mu_{\hat{G}}(x)^{j}, \mu_{\hat{G}}(y)^{j}\right\}
\end{aligned}
$$

FGI is satisfied

$$
\begin{aligned}
(\mathrm{FG} 2) \quad\left(\mu_{\hat{G i} \cap \hat{\mathrm{Gj}}}\right)(\mathrm{x}) & =\min \left\{\mu_{\hat{\mathrm{Gi}}}(\mathrm{x}), \mu_{\hat{\mathrm{Gj}}}(\mathrm{x})\right\} \\
& =\min \left\{\mu_{\hat{\mathrm{Gi}}}\left(\mathrm{x}^{-1}\right), \mu_{\mathrm{Gi}}\left(\mathrm{x}^{-1}\right)\right. \\
(\mathrm{FG} 3) \mu_{\hat{\mathrm{Gsi}} \cap \hat{\mathrm{G}}_{\mathrm{j}}(\mathrm{e})} & =\min \left\{\mu_{\hat{\mathrm{Gi}}}(\mathrm{e}),\left\{\mu \hat{\mathrm{G}}_{\mathrm{j}}(\mathrm{e})\right\}\right. \\
& =\min \{1,1) \\
& =1
\end{aligned}
$$

Similarly we follow the above conclusion in the cases of $\mathrm{i}<\mathrm{j}$ and $\mathrm{i}=\mathrm{j}$.

Hence $\mathrm{Gi} \cap \mathrm{Gj}$ is also a fuzzy group for all natural numbers $\mathrm{i}$ and $\mathrm{j}$.

2.7 Proposition : Let $<A>=\left\{A, A^{1}, A^{2},-----A^{P}--\right.$ --E\} then $U A^{P}=A$ and $\cap A^{P}=E$ where $\mathrm{p}=1$ to $\infty$.

Proof: Let $\mathrm{x} \varepsilon \mathrm{G}$ we have $\mathrm{A} \subset \mathrm{U} \mathrm{A}^{\mathrm{P}}$---- (i)

$$
\begin{aligned}
& \left(\text { since } \mathrm{A}(\mathrm{x}) \leq \mathrm{UA}^{\mathrm{p}}(\mathrm{x})\right) \\
& \begin{aligned}
\mathrm{UA}^{\mathrm{P}}(\mathrm{x}) & =\max \left\{\mathrm{A}(\mathrm{x}), \mathrm{A}^{2}(\mathrm{x}),-----\right\} \\
& =\mathrm{A}(\mathrm{x}) \quad\left(\text { since } \mathrm{A}(\mathrm{x}) \leq(\mathrm{A}(\mathrm{x}))^{\mathrm{p}}\right. \text { for all }
\end{aligned} \\
& \text { natural number } \mathrm{p} \text {. }
\end{aligned}
$$


$\mathrm{U} \mathrm{A}^{\mathrm{p}} \subset \mathrm{A}$ (ii) from (i) and (ii)

$\mathrm{A}^{\mathrm{p}}=\mathrm{A}$. Also we have to

Show that $E=\cap A^{p}$. Let e $\varepsilon A^{P}$ implies that $(e, 1) \varepsilon$ $\mathrm{A}^{\mathrm{p}}$ for all $\mathrm{p}$.

Implies that $(\mathrm{e}, 1) \cap \mathrm{A}^{\mathrm{p}}$

Therefore $\mathrm{E} \subset \cap \mathrm{A}^{\mathrm{p}}$ (iii) Let $\mathrm{x} \varepsilon$

$\cap \mathrm{A}^{\mathrm{p}}$ implies that $\mathrm{x} \varepsilon \mathrm{A}^{\mathrm{p}}$ for all $\mathrm{p}$.

$$
\begin{aligned}
\cap \mathrm{A}^{\mathrm{P}}(\mathrm{x}) & =\min \left\{\mathrm{A}(\mathrm{x}), \mathrm{A}^{2}(\mathrm{x})-----\right\} \\
& = \begin{cases}0 & \text { if } \mathrm{x} \neq \mathrm{e} \\
1 & \text { if } \mathrm{x}=\mathrm{e}\end{cases}
\end{aligned}
$$

$\mathrm{x} \varepsilon \cap \mathrm{A}^{\mathrm{p}}$ implies that $\mathrm{x}=\mathrm{e}$ thus $\cap \mathrm{A}^{\mathrm{p}} \subset \mathrm{E}$ (iv) hence

From (iii) and (iv) $\mathrm{E}=\cap \mathrm{A}^{\mathrm{P}}$

2.8 Definition : Let $\mathrm{f}: \mathrm{G} \rightarrow \mathrm{G}^{1}$ be a homomorphism's of groups. For any fuzzy set A $\varepsilon$ $G^{1}$ we define a new fuzzy set $A^{f} \varepsilon G$ by $A^{f}(x)=$ Af(x) for all $x \in G$.

2.9 Proposition: Let $G$ and $G$ ' be groups and ' $f$ 'be homomorphism from $G$ onto $G^{1}$, (i) if $A$ is fuzzy group of $G^{1}$ then $A^{f}$ is fuzzy group of $G$. (ii) if $A^{f}$ is fuzzy group of $G$ then $A$ is fuzzy group of $G^{1}$.

Proof: (i) Let $\mathrm{x}, \mathrm{y} \varepsilon \mathrm{G}$, we have

$$
\begin{aligned}
(\mathrm{FG} 1) \mathrm{A}^{\mathrm{f}}((\mathrm{xy})) & =\mathrm{A}(\mathrm{f}(\mathrm{x}), \mathrm{f}(\mathrm{y})\} \\
& \geq \min \{\operatorname{Af}(\mathrm{x}), \operatorname{Af}(\mathrm{y})\} \\
& =\min \left\{\mathrm{A}^{\mathrm{f}}(\mathrm{x}), \mathrm{A}^{\mathrm{f}}(\mathrm{y})\right\} \\
(\mathrm{FG} 2) \mathrm{A}^{\mathrm{f}}(\mathrm{x}) & =\mathrm{A}(\mathrm{f}(\mathrm{x})) \\
& =\mathrm{A}\left(\mathrm{f}(\mathrm{x})^{-1}\right) \\
& =\operatorname{Af}\left(\mathrm{x}^{-1}\right)=\mathrm{A}^{\mathrm{f}}\left(\mathrm{x}^{-1}\right) \text {.Therefore } \mathrm{A}^{\mathrm{f}} \text { is }
\end{aligned}
$$

fuzzy group of $\mathrm{G}$.

(ii) for any $\mathrm{x}, \mathrm{y} \varepsilon \mathrm{G}$, there exists a,b $\varepsilon \mathrm{G}$ such that $\mathrm{f}(\mathrm{a})=\mathrm{x}$ and $\mathrm{f}(\mathrm{b})=\mathrm{y} \cdot(\mathrm{FG} 1) \mathrm{A}((\mathrm{xy}))=\mathrm{A}(\mathrm{f}(\mathrm{a})$, $\mathrm{f}((\mathrm{b})\}$

$$
\begin{aligned}
& =A^{f}((a b)) \\
& \geq \min \left\{A^{f}(a), A^{f}(b)\right\} \\
& =\min \{\operatorname{Af}(a), \operatorname{Af}(b)\} \\
& =\min \{A(x), A(y)\}
\end{aligned}
$$

Volume 6-No.3, September 2010

(FG2) $A\left(x^{-1}\right)=A(f(a))^{-1}=\operatorname{Af}\left(a^{-1}\right)=A^{f}\left(a^{-1}\right)=A^{f}(a)$ $=\mathrm{A}(\mathrm{x})$.

2.10 Proposition: Let $G$ and $G^{1}$ be groups and $f$ is homomorphism from $G$ onto $G^{1}$. If $A^{f}$ is fuzzy group of $G$, then $A$ is fuzzy group of $G^{1}$.

Proof: By proposition (2.9), A is fuzzy group of $\mathrm{G}^{1}$. for any $\mathrm{y} \varepsilon \mathrm{G}^{1}$.

$$
\begin{aligned}
& \text { (FG1) } A^{f}(y)=\operatorname{supA}(\mathrm{z}) \geq \sup A(\mathrm{x}) \geq \\
& \mathrm{z} \& \mathrm{f}^{-1}(\mathrm{y}) \quad \mathrm{f}(\mathrm{x})=\mathrm{y} \\
& \sup A(x)=\quad A^{f}(y) \\
& \mathrm{f}(\mathrm{x})=\mathrm{y} \\
& (\mathrm{FG} 2) \mathrm{A}^{\mathrm{f}}\left(\mathrm{x}^{-1}\right)=\operatorname{supA}(\mathrm{z})=\operatorname{supA}(\mathrm{x}) \\
& z \& f^{-1}(y) f(x)=x^{-1}
\end{aligned}
$$

where $x \in f^{-1}(y)$

$$
\begin{aligned}
& =\sup A(x)=\sup A(x) \\
& \begin{array}{l}
f(x)=x^{-1} \quad f(x)=x^{-1} \\
\geq \sup A\left(x^{-1}\right) \\
\quad f(x)=x^{-1} \\
=A^{f}\left(x^{-1}\right) \text { A is fuzzy group of } G^{1} .
\end{array}
\end{aligned}
$$

2.11 Definition : Let A be a M- fuzzy group, then the following set of fuzzy groups $\left\{\mathrm{A}, \mathrm{A}^{1}\right.$, $\left.\mathrm{A}^{2}, \ldots . \mathrm{A}^{\mathrm{p}} \ldots \mathrm{E}\right\}$ is called fuzzy group family generated by A. It will be denoted by $\langle\mathrm{A}\rangle$.

2.12 Proposition : Let A be a fuzzy group, then A $\supset \mathrm{A}^{2} \supset \mathrm{A}^{3} \ldots \supset \mathrm{A}^{\mathrm{p}} \ldots \mathrm{E}$.

Proof: It is known that $\mathrm{A}(\mathrm{a}) \varepsilon[0,1]$, hence $\mathrm{A}(\mathrm{a}) \geq$ $\mathrm{A}(\mathrm{a})^{2}, \mathrm{~A}\left(\mathrm{a}^{2}\right) \geq\left(\mathrm{A}\left(\mathrm{a}^{2}\right)\right)^{2}, \ldots \mathrm{A}\left(\mathrm{a}^{\mathrm{n}}\right) \geq\left(\mathrm{A}\left(\mathrm{a}^{\mathrm{n}}\right)\right)^{2}$ by using the definition of fuzzy subsets, this gives that $\mathrm{A} \supset$ $\mathrm{A}^{2}$. By generalizing it for any natural numbers $i$ and $\mathrm{j}$ with $\mathrm{i} \leq \mathrm{j}$. we obtain $\left(\mathrm{A}_{\mathrm{i}}(\mathrm{a})\right)^{\mathrm{i}} \geq\left(\mathrm{A}_{\mathrm{j}}(\mathrm{a})\right)^{\mathrm{j}}$, $\left(A_{i}\left(a^{2}\right)\right)^{i} \geq\left(A_{j}\left(a^{2}\right)^{j}, \ldots \ldots . .\left(A_{i}\left(a^{n}\right)\right)^{i} \geq A_{j}\left(a^{n}\right)\right)^{j}$.

So $A^{i} \supset A^{j}$ for any natural numbers $i$ and $j$ with $i \leq j$ which means that $\mathrm{A} \supset \mathrm{A}^{2} \supset \mathrm{A}^{3} \ldots . \supset \mathrm{A}^{\mathrm{pn}} \ldots$ finally we get $\mathrm{E}=\cap \quad \mathrm{A}^{\mathrm{p}}$ which is immediate from proposition (2.7). Since

$\mathrm{Lt} \quad \mathrm{A}(\mathrm{a})^{\mathrm{p}}=1 \quad$ if $\mathrm{a}=\mathrm{e}$. 
$\mathrm{n} \rightarrow \infty \quad=0 \quad$ if $\mathrm{a} \neq \mathrm{e} \quad$ we then obtain the required relations.

\section{L-FUZZY NUMBER}

A fuzzy number is a fuzzy set on the real axis with convex, normal and continuous. (ie) A mapping $\lambda$ : $\mathrm{R} \rightarrow[0,1]=\mathrm{L}$ associating with each real number ' $\mathrm{t}$ ', its grade of membership $\lambda(t)$. A fuzzy number ' $\lambda$ ' is called convex if $\lambda(\mathrm{t}) \geq \min \{\lambda(\mathrm{s}), \lambda(\eta)\}, \mathrm{s} \leq \mathrm{t} \leq \eta, \mathrm{s}, \mathrm{t}$, $\eta \varepsilon R$. If ' $\lambda$ ' is normal then there exists $t_{0} \varepsilon R$ such that $\lambda($ to $)=1$.

A fuzzy number ' $\lambda$ ' will be called upper semi continuous provided for all teR with $\lambda(\mathrm{t}) \leq \alpha$, there is $\delta>0$ such that $|\mathrm{s}-\mathrm{t}| \leq \delta \rightarrow \lambda(\mathrm{s}) \leq \alpha$. The fuzzy real line $R_{L}$ consists of 1-fuzzy numbers which fulfill regularity condition. If $\mathrm{R}_{\mathrm{L}}$ denote the set of all regular L-fuzzy numbers, then RL will be called the L-fuzzy real line.A fuzzy number ' $\lambda$ ' is called non-negative if $\lambda(t)=0$. The equality of fuzzy number ' $\lambda$ ' and ' $\mu$ ' is defined by $\lambda=\mu$ iff $\lambda(\mathrm{t})=\mu$ (t) for al tcR. The arithmetic operation,,$+- \bullet$ and /on $\mathrm{R}_{\mathrm{L}} \times \mathrm{R}_{\mathrm{L}}$ are defined by

(i) $(\lambda+\mu)(\mathrm{t}) \quad=\mathrm{V}_{\mathrm{S} \varepsilon \mathrm{R}}\{\min (\lambda(\mathrm{s}), \mu(\mathrm{t}-\mathrm{s})\}$

$\mathrm{t} \varepsilon \mathrm{R}$.

(ii) $(\lambda-\mu)(\mathrm{t}) \quad=\mathrm{V}_{\mathrm{S} \varepsilon \mathrm{R}}\{\min (\lambda(\mathrm{s}), \mu(\mathrm{s}-\mathrm{t})\}$

$\mathrm{t} \varepsilon \mathrm{R}$.

(iii) $(\lambda \mu)(\mathrm{t}) \quad=\mathrm{V}_{\mathrm{S} \varepsilon \mathrm{R}}\{\min (\lambda \mathrm{s}), \mu(\mathrm{t} / \mathrm{s})\}$

$\mathrm{t} \varepsilon \mathrm{R}$.

Additive and multiplicative identities in $\mathrm{R}_{\mathrm{L}}$ are and

$\begin{aligned} \mathrm{O}(\mathrm{t}) & \begin{cases}1 & \text { if } \mathrm{t}=0 \\ 0 & \text { if } \mathrm{t} \neq 1\end{cases} \\ \mathrm{I} & \begin{cases}1 & \text { if } \mathrm{t}=1 \\ 0 & \text { if } \mathrm{t} \neq 1\end{cases} \end{aligned}$

the operations + and - are associative and commutative with the identities 0 and 1 respectively. The level set of sum, different and product fulfill the following conditions. $[\lambda+\mu]=[\lambda]_{\alpha}+[\mu]_{\alpha}$

$[\lambda+\mu]=[\lambda]_{\alpha}+[\mu]_{\alpha}$. In general, it is not oblivious that for $\mathrm{f}: \mathrm{X} \times \mathrm{Y} \rightarrow[0,1]$ and $\lambda, \mu$ fuzzy subsets of $\mathrm{X}$ and $\mathrm{Y}$ respectively. We would always have $\mathrm{f}\{(\lambda, \mu)\}=\mathrm{f}\left\{[\lambda]_{\alpha},[\mu]_{\alpha}\right\}$ for all $\alpha \varepsilon \mathrm{L}$. However it may be shown that if $\mathrm{L}=[0,1]$ and $\mathrm{f}: \mathrm{R} \times \mathrm{R} \rightarrow \mathrm{R}$ be continuous, then $\mathrm{f}[(\lambda, \mu)]_{\alpha}=\mathrm{f}\left[[\lambda]_{\alpha},[\mu]_{\alpha}\right.$ for all $\alpha \varepsilon$ $\mathrm{L}$ and all upper semi continuous L-fuzzy sets $\lambda$ and Hon R. A fuzzy number ' $\lambda$ ' is regular if

(i) $\lambda$ is convex

(ii) $\lambda$ is normal

(iii) $\lambda$ is upper semi continuous.

(iv)Each level of $\lambda$ is bounded.

3.1Proposition : ' $\lambda$ ' is fuzzy number with convex function. Then $\lambda$ is constant function.

Proof $: \lambda: R \rightarrow[0,1]$ is a map let $a, b \varepsilon R$ and if $a \leq$ b. Let $\beta \varepsilon \mathrm{R}$ such that $\beta \leq \mathrm{a}$ (ie) $\alpha$ is convex function, we get

$\mathrm{a} \leq \alpha \leq \mathrm{b}$ implies $\lambda(\alpha) \geq \lambda(\mathrm{a})$

$$
\lambda(\alpha) \geq \lambda(b)
$$

$\mathrm{a} \leq \mathrm{b} \leq \mathrm{b}+\alpha$ wehave $\lambda(\mathrm{b}) \geq \lambda(\mathrm{a})$

$\beta \leq \mathrm{a} \leq \mathrm{b} \quad$ so that $\lambda(\mathrm{a}) \geq \lambda(\mathrm{b})$

$\mathrm{a} \leq \mathrm{b} \leq \mathrm{b}+\alpha$ hence

$\lambda(\mathrm{b}) \geq \lambda(\alpha) \quad$ implies

$\lambda(\alpha)=\lambda($ b) by (ii) and (iv) we conclude that

$\lambda(\mathrm{x})=\lambda(\mathrm{b})$ for all $\mathrm{x} \varepsilon(\mathrm{a}, \mathrm{b})$

$=\lambda(\mathrm{a}) . \mathrm{a} \geq \mathrm{b}$ is following in a similar way.

Let $\beta \varepsilon \mathrm{R}$ such that $\beta \leq \mathrm{a} \leq \mathrm{b}$ by $\beta \leq \mathrm{a}, \lambda(\beta)=\lambda(\mathrm{a})$

Let $\gamma \varepsilon \mathrm{R}$ such that $\mathrm{a} \leq \mathrm{b} \leq \gamma$, by $\mathrm{b} \leq \gamma$ implies $\lambda$ (b) so that $\lambda(\gamma)$ we conclude that

$$
\lambda(\mathrm{a})=\lambda(\mathrm{b}) \text { for all } \mathrm{a}, \mathrm{b} \varepsilon \mathrm{R}
$$

$\lambda$ is a constant function.

3.2 Proposition : Let a fuzzy number ' $\lambda$ ' be a convex function. Then $(\mathrm{R},+, \mathrm{O})$ is a fuzzy group.

Proof : ' $\lambda$ ' is a fuzzy number and convex function by (3.1), $\lambda$ is a constant function. 
(FG1) Let $\mathrm{x}, \mathrm{y} \varepsilon \mathrm{R}$, we have $\lambda(\mathrm{x})=\mathrm{C}$,

$$
\begin{aligned}
\lambda(\mathrm{xy})=\mathrm{C} & \lambda(\mathrm{y})=\mathrm{C} \\
= & \min \{\lambda(\mathrm{x}), \lambda(\mathrm{y})\}
\end{aligned}
$$

(FGI) is satisfied in $\mathrm{R}$

(FG2) $\lambda(\mathrm{x})=\mathrm{C}$ for all $\mathrm{x} \varepsilon \mathrm{R}$

$$
\lambda(\mathrm{x})=\lambda(-\mathrm{x})
$$

FG2 is satisfied in $R$.

Conclusion: Dib.K.A[4] and A. Rosenfeld [7] introduced the concept of fuzzy normal subgroups and fuzzy groups. In this paper we investigate the concept of fuzzy groups and obtain some Results. One can obtain these results by applying two fuzzy membership functions.

Application: Group theory has many applications in Physics, Chemistry and Computer science problem.

Acknowledgement: The authors are highly thankful to the referees for their valuable comments and suggestions for improving the paper. This research was partially supported by J.J EDUCATIONAL HEALTH \& CHARITABLE TRUST, TRICHIRAPPALLI-09.
International Journal of Computer Applications (0975 - 8887)

Volume 6-No.3, September 2010

\section{References:}

[1] Antony, J.M and Sherwood, H., Fuzzy groups redefined, J.Math.Anal.Appl.69,124130,1979.

[2] Deminci. M, Smooth subgroups and Smooth Homomorphism's, Fuzzy set and systems, 117, 439446, 2001.

[3] Dobrista, V.P and Yahhyaeva, G.R., On Homomorphism's of fuzzy groups, Siberian mathematical journal, 43,47-51,2002.

[4] Rosenfeld, A. Fuzzy groups, Journal of mathematical Analysis andApplications, 35, 512$517,1971$.

[5] A.Solairaju and R.Nagarajan Structure Properties of M-fuzzy groups, Accepted For Publications Applied Mathematical Sciences, 2010.

[6] Zadeh,L.A., Fuzzy sets, Inform and control, 8 , 338-353, 1965.

[7] Zadeh.L.A., Fuzzy sets and systems, Proc.Symp.Syst.Theory. Polytech.Inst.Brooklyn PP 29-37, 1965. 